\title{
Chronic interstitial lung disease in children
}

\author{
Maria Aparecida S. S. Paiva,1 Sandra M. M. Amaral2
}

\begin{abstract}
Objectives: To describe clinical and diagnostic features and the results of therapeutic conduct in a group of pediatric patients with chronic interstitial lung disease.

Methods: A retrospective study of 25 immunocompetent patients, aged 2 months to 17 years, with chronic interstitial lung disease, admitted to the Pediatric Pulmonary Section, Department of Pediatrics, Hospital dos Servidores do Estado, over a 20-year period (1984-2004). A routine protocol for persistent chronic pneumonias was used and the patients with interstitial lung disease were selected. Clinical, laboratory and imaging data were analyzed.

Results: Twenty-five patients were diagnosed with chronic interstitial lung disease, 13 were aged less than 2 years and 17 were male. Diagnoses were made based on history, physical examination and routine tests in one case, based on more complex tests in three cases and based on the results of invasive tests in 21 cases ( 20 by lung biopsy and one by bronchoalveolar lavage). Except for one patient with pulmonary lymphangiectasia, the long-term treatment ( 1 to 7 years) consisted of corticosteroid, in six cases associated with hydroxychloroquine. Four patients required home oxygen therapy. The authors followed the patients in the outpatient department ( 6 a 8 visits/year). Patient outcome was: good (15); regular, with mild sequelae (4); and poor, with severe sequelae (3). One patient was lost in the follow-up period and two died.

Conclusions: Chronic interstitial lung diseases in children are a group of rare pulmonary disorders, but a relevant one because of the possible progression to pulmonary fibrosis. Early diagnosis and a long-term, specialized treatment and follow-up are important for the patient outcome. Pediatricians should be aware of these diseases because in many cases diagnosis and treatment are overlooked.
\end{abstract}

J Pediatr (Rio J). 2007;83(2):233-240: J Pediatr (Rio J). 2007;83(3):233-240: Chronic interstitial lung diseases, children.

\section{Introduction}

The large group of interstitial lung diseases in children includes a subgroup of rare chronic lung diseases that are characterized by diffuse pulmonary infiltrate, restrictive functional changes, hypoxemia and failure to thrive. In the most advanced clinical forms, chest deformities, digital clubbing and cardiac involvement with signs of cor pulmonale can all be present. This is a heterogenous group of diseases, which etiologies are sometimes unknown, involving alterations in the alveolar spaces and distal airways, in addition to alveolar wall thickening. Knowledge of these diseases is of clinical relevance due the high rates of morbidity and mortal- ity. Furthermore they may progress to pulmonary fibrosis. The differential diagnosis is a challenge, involving more than a hundred of causes. ${ }^{1-3}$

These diseases remain difficult to manage, since the small number of cases is a factor that limits the possibility of clinical studies and research. ${ }^{4,5}$

The need for an internationally standardized nomenclature and diagnoses led to the establishment of a classification, drawn up by a multidisciplinary group from the American Thoracic Society/European Respiratory Society (ATS/ERS), in a consensus process concluded in 2001 , and emphasizing

1. Setor de Pneumologia Pediátrica, Hospital dos Servidores do Estado, Rio de Janeiro, RJ, Brasil. Coordenadora, Centro de Diagnóstico de Doenças Respiratórias, Clínica Pediátrica, Centro Médico Barrashopping, Rio de Janeiro, RJ, Brasil. Comitê de Doenças do Aparelho Respiratório, Sociedade de Pediatria do Estado do Rio de Janeiro (SOPERJ), Rio de Janeiro, RJ, Brasil.

2. Setor de Pneumologia Pediátrica, Hospital dos Servidores do Estado, Rio de Janeiro, RJ, Brasil.

Manuscript received June 08 2006, accepted for publication Jan 172007.

Suggested citation: Paiva MA, Amaral SM. Chronic interstitial lung disease in children. J Pediatr (Rio J). 2007;83(3):233-240.

doi 10.2223/JPED.1635 
the importance of clinico-radiologic-pathologic interaction when studying these diseases. ${ }^{6}$ Figure 1 is a modified representation of that consensus. For the purposes of the present paper, the childhood forms described were included, such as pulmonary glycogenosis, ${ }^{7,8}$ chronic pneumonitis of infancy, ${ }^{9}$ persistent tachypnea of infancy with neuroendocrine cell hyperplasia ${ }^{7,10}$ and genetic surfactant abnormalities. ${ }^{7,11}$

We could not find any Brazilian publication on a series of patients with chronic interstitial lung diseases in the pediatric age group.

The aim of this paper is to describe the clinical and diagnostic features and the results of therapeutic conduct in a group of pediatric patients with these diseases. through the patient admission records system, completed systematically by the medical team and containing data on demographics, initial and final diagnosis. There are additional data from a protocol for chronic lung diseases used during diagnosis and follow-up. The information collected from the protocol and medical records includes clinical, laboratory, radiological and functional data, in addition to diagnosis and outcome.

Patients were referred to our section by other hospitals, by our own pneumology outpatient department and other sections of the Department of Pediatrics that request consultations with us. Inclusion criteria were age ( $<18$ years), presence of dyspnea (tachypnea and/or respiratory dis-

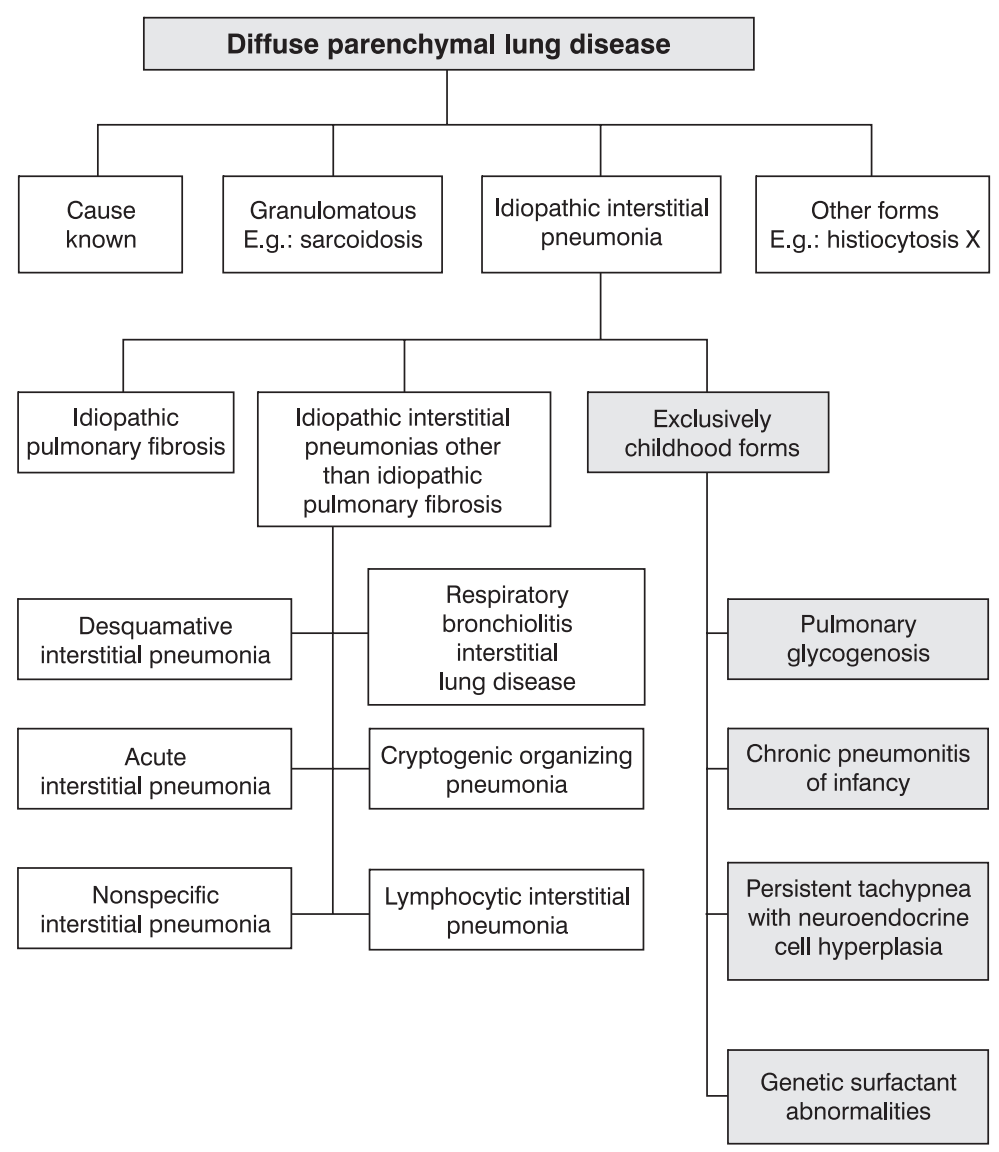

Figure 1 - Consensus of the American Thoracic Society/European Respiratory Society ${ }^{6}$ with the addition of exclusively childhood forms $\mathrm{s}^{7-11}$

\section{Methods}

We have assessed our experience with chronic interstitial lung disease (ILD) in the form of an observational descriptive study of 25 patients admitted to the Pediatric Pulmonary Section, Department of Pediatrics, Hospital dos Servidores do Estado (HSE). The patients were diagnosed and followed over a 20-year period (January 1984 to January 2004). This convenience sample contains all consecutive cases identified tress), crackles or diffuse pulmonary interstitial images and the persistence of any of these findings for a minimum period of 1 month. ${ }^{12}$ Patients were excluded if they had immunodeficiencies, cystic fibrosis, bronchopulmonary dysplasia, obliterative bronchiolitis or neoplasms.

In the history, we emphasized the severity and progressive nature of dyspnea and failure to thrive. In infants, 
respiratory distress was observed during feeding or crying, characteristic of exertional dyspnea. Questions were asked about the presence of cough and its characteristics (dry or productive, hemoptoic sputum). The questionnaire also contained questions about any history of previous or recurrent infection, hospital admissions due to lung disease, environmental and family history, medication, symptoms suggestive of aspiration syndrome and related to systemic diseases, such as joint, skin, kidney and nervous system diseases.

In the physical examination, we emphasized the following features, due to their relevance: fever, pallor, cyanosis, tachypnea, signals of respiratory distress (retractions, grunting and/or nasal flaring), "velcro-like" crackles, wheezing, malnutrition (weight below 90\%), chest deformities with flattening of the anteroposterior diameter, finger clubbing and signs of pulmonary hypertension, and/or cor pulmonale (loud second heart sound, tachycardia, galloping rhythm, jugular venous distension, hepatomegaly and edema of the lower limbs).

Guided by history and physical examination, we sequentially chose the tests indicated, ${ }^{13}$ starting with non-invasive methods, such as blood tests, arterial blood gas analysis at rest and, when possible, after exercise, radiology, serology for HIV and cytomegalovirus, tests for congenital infections in infants, immunological profile, investigation for aspiration syndromes and collagenosis, sweat test, cardiology assessment and, for older patients, respiratory function test. Conventional radiology was used regularly, but high resolution computerized tomography (HRCT) was not available at our hospital for the initial cases. Pulmonary perfusion scintigraphies were indicated for assessing severity and, when possible, 67-gallium scintigraphy was used in order to detect the presence of alveolitis.

Subsequently, invasive tests were ordered, together with the tests to be run on the material obtained. Bronchoalveolar lavage (BAL) was not performed as a routine procedure for assessing cellularity, since it requires children to be sedated and increases the potential risk of aggravating hypoxemia. It was indicated when there was a suspicion of diseases such as pneumonia due to mineral oil aspiration, hemosiderosis, alveolar proteinosis and to investigate infectious agents, thereby avoiding a biopsy in one case.

Lung biopsy was performed when a specific diagnosis could not be achieved through the methods described above. Later on, histopathological test results were reviewed in the light of the 2001 ATS/ERS consensus classification.

Treatment was in accordance with guidelines in the literature for the management of inflammation, with prednisone 1 to $2 \mathrm{mg} / \mathrm{kg}$ per day, in a single morning dose for 3 to 6 months, reducing to half the dosage for another 6 months, and then long-term treatment varying from 1 to 7 years, generally on alternate days once a sustained improvement has been achieved. ${ }^{2,14,15}$ We used hydroxychloroquine in daily doses of $10 \mathrm{mg} / \mathrm{kg}$ when response to the corticosteroid was poor or when significant side-effects were observed, aiming to reduce or withdraw it. ${ }^{14-16}$ For one patient the choice was pulse therapy (methylprednisolone $-10 \mathrm{mg} / \mathrm{kg} / \mathrm{day}, 3$ days /month, 6 months) because of side effects from oral corticosteroid therapy. The response and tolerance were good. ${ }^{14,17}$ Side effects were monitored and doses were adjusted or the regimen altered, and we did not observe any situation in which treatment could not be prescribed. When we were able to define etiology we adopted specific measures. Other therapeutic measures included, when necessary, oxygen therapy, ${ }^{18}$ nutritional support, blood transfusion, respiratory physiotherapy and psychological support.

Outcome was defined as good when the patient exhibited no limitations to physical activity; regular, with mild sequelae, when dyspnea occurred in response to moderate or heavier effort; and poor, with severe sequelae if there was dyspnea in response to mild effort.

\section{Results}

The 25 patients were aged from 2 months to 17 years (mean of 34 months and median of 19 months); 13 aged less than 2 years; and 17 being male (Table 1 ). In the cases of two patients, we found another ILD case in the same family (mother and brother).

Clinical presentation was varied. In Table 2 we have selected the data from history and physical examination that we consider of greatest importance in these diseases, although the admission protocols covered a complete physical examination. Eleven children exhibited clinical signs of pulmonary hypertension with a loud second heart sound, confirmed by electrocardiogram (ECG) and echocardiogram. In just one patient (17 years), with desquamative interstitial pneumonia and accentuated fibrosis, there were clinical signs of heart failure. Hypoxemia (saturation < 90\%) was confirmed in 22 patients and polycythemia in 14. Polycythemia was correlated with more severe and prolonged hypoxemia.

We managed to make a diagnosis without lung biopsy in five patients. We diagnosed hypersensitivity pneumonitis based entirely on a highly suggestive history and simple tests in one patient, with other hypotheses ruled out later on, and with good response to changed environment and antiinflammatory treatment. Lung biopsy was performed in 20 cases, in 18 by thoracotomy and in two by videothoracoscopy with a quicker recovery. There were no unfavorable events during these procedures, even in the patients with hypoxemia, two of them were on mechanical ventilation. Final diagnosis and the method of definition are shown in Table 3.

Twenty four patients were treated with corticosteroids (one received pulse therapy) and six with hydroxychloroquine. Patients with pulmonary silicosis and hypersensitivity pneumonia were isolated from the environment, in addition to receiving antiinflammatory treatment. Two children underwent surgery for gastroesophageal reflux, one also had severe pectus excavatum corrected surgically. In the case of the patient with pulmonary lymphangiectasia, pleural punc- 
ture was performed and a special diet prescribed. Twenty-two patients required oxygen therapy at the start of treatment for a variable period. Four were put on home oxygen for periods of 6 months to 2 years, and 11 were given pharmacological treatment for pulmonary hypertension.

We observed good outcome in 15 patients (60\%), with a return of development, good quality of life and no exertional dyspnea, while four exhibited regular outcome, with mild sequelae. One patient with a chronic aspiration syndrome that took more time to be diagnosed and had severe pectus excavatum, and another with pulmonary hemosiderosis developed pulmonary fibrosis and, although they improved, still have poor prognosis, and were still being monitored at the time of writing. One patient with lymphocytic interstitial pneumonia (LIP) complied irregularly with treatment, which was suspended later on, due to a maternal decision. This patient presented a relapse, and the treatment was restarted recently, but there were severe sequelae and images suggestive of fibrosis and honeycombing on HRCT. We recorded two deaths. One patient with desquamative interstitial pneumonia (DIP), pulmonary fibrosis and cor pulmonale, since the first presentation, died after 6 years of improvement in response to treatment. The other patient, diagnosed with pulmonary hemosiderosis, died in her home town after being treated successfully for 10 months. One patient abandoned follow-up before treatment was complete, but with a good initial response. None of them exhibited outcome suggestive of kidney disease or collagenosis during follow-up.

\section{Discussion}

The Department of Pediatrics at Hospital dos Servidores do Estado do Rio de Janeiro is divided into sections, and has a records system covering all patients who are admitted. Each section has specific records.

Two hundred and sixty patients/year are admitted to the Pneumology Section and an average of 1,140 outpatients /year are assessed and followed by the pediatric pulmonologists. Patients with acute or chronic pulmonary diseases who do not require admission to the intensive care unit (ICU) are admitted to the specialized ward. The patients are checked daily by the doctors (pulmonologists and residents) and followed by the same specialist in the outpatient setting allowing us to observe their outcome personally. All cases are recorded by the medical team. Our section also assesses patients from other sections who develop severe or persistent respiratory problems. It is possible that an occasional

Table 1 - Demographic data for 25 patients with chronic interstitial lung disease

\begin{tabular}{ll}
\hline Variable & Number of patients \\
\hline Sex & 8 \\
Female & 17 \\
Male & \\
Race & 17 \\
White & 4 \\
Black & 4 \\
Mixed & \\
Age & 9 \\
$\leq 1$ year & 4 \\
$>1-2$ years & 9 \\
$>2-6$ years & 2 \\
$>6-10$ years & 1 \\
$>10$ years & \\
&
\end{tabular}


patient with ILD may have died or been discharged, mainly from the ICU and nursery, without being assessed by the pulmonologists and without being diagnosed, but it is likely that such loss is small due to the structure of the Pediatric Department and the rarity of the disease.

It should be pointed out that long-term retrospective studies have their limitations. The quality of our data is guaranteed by the records system and the protocol that is filled out as each case is dealt with. Other limitations are related to certain unavailable diagnostic methods, particularly during the initial phase. However, we consider this study a relevant series since there are no Brazilian data published to date.

Our study supports the position that ILD are a heterogenous group of rare diseases that should be given prominence within the group of chronic pulmonary diseases because they require referral to specialist centers with the necessary resources to investigate them.

We assessed our patients and compared them with some published series. ${ }^{15,19,20}$ The use of a routine approach for history and sequential tests made possible establish a diagnosis that was highly suggestive or specific in all the cases. According to published data, 13 patients were less than 2 years old ${ }^{15}$ and six presented with symptoms during their first month of life. We routinely perform investigations for ILD in full term or close to term newborn infants who present persistent cough or have problems of being weaned off mechanical ventilation, without evidence of infectious diseases.

Failure to thrive was a relevant factor in history, but it was not a spontaneous complaint in most of the cases. The same was true of exertional dyspnea, so parents should be asked

Table 2 - Signs and symptoms of presentation of 25 patients with chronic interstitial lung disease

\begin{tabular}{|c|c|}
\hline & Number of patients \\
\hline \multicolumn{2}{|l|}{ Symptoms } \\
\hline Failure to thrive & 23 \\
\hline Dyspnea at rest & 19 \\
\hline Dry cough & 14 \\
\hline Cyanosis & 13 \\
\hline Recurrent infections & 10 \\
\hline Wheezing & 9 \\
\hline Fever & 8 \\
\hline Dyspnea only after exercise & 5 \\
\hline Productive cough & 5 \\
\hline Signs & 21 \\
\hline Tachycardia or gallop & 19 \\
\hline Under development & 19 \\
\hline Tachypnea & 19 \\
\hline Finger clubbing & 15 \\
\hline Pallor & 13 \\
\hline Chest deformities & 12 \\
\hline Loud S2 & 11 \\
\hline Wheezing/crackles & 11 \\
\hline Jugular venous distension & 1 \\
\hline
\end{tabular}


about these symptoms. Tachypnea, present in 19 of our patients, is considered the main symptom and is often the first and only clinical manifestation. ${ }^{3}$ In 22 cases, patients had crackles, but pulmonary auscultation may be normal, as was the case of three patients. Wheezing is less common but it was observed in 11 patients.

In the 13 critically ill patients, there was cyanosis, and two patients were on mechanical ventilation when the diagnosis was suspected. In a statistical analysis of the signs and symptoms of 99 patients, Fan et al. ${ }^{12}$ established a severity "score", in which the degree of hypoxemia and pulmonary hypertension were the severity factors best related to prognosis. We observed the same in our series, but further studies are needed.

Conventional radiography can identify interstitial infiltrate, generally bilateral, but may also be normal in around
$10 \%$ of ILD cases in adults. This was the case with one of our patients, but the presence of cyanosis on exertion motivated investigation. In 1994, HRCT was introduced as the most important imaging technique for diagnosis and management of ILD in children and, in our hospital, became available only in 1997. As a result, in five cases HRCT scans were not performed and seven were only scanned after diagnosis. Among the other 13 patients who were scanned, we initially found in the scans a predominance of ground glass images, guiding the lung biopsy location. Two patients developed honeycombing. Recently, the artifacts produced in HRCT by tachypnea in infants have been avoided with a new method described by Long et al. ${ }^{21}$ and known as controlledventilation HRCT, which allows respiratory function to be studied concomitantly. We performed perfusion scintigraphy in 12 patients, finding variable degrees of hypoperfusion,

Table 3 - Final diagnosis for the 25 patients with chronic interstitial lung disease and method of diagnosis

\begin{tabular}{|c|c|c|}
\hline Diagnosis & Method & Number of patients \\
\hline LIP & Biopsy & 8 \\
\hline DIP & Biopsy & 4 \\
\hline NSIP & Biopsy & 4 \\
\hline COP & Biopsy & 1 \\
\hline Silicosis & Biopsy & 1 \\
\hline Hemosiderosis & Biopsy & 1 \\
\hline Aspiration syndrome/GER & Biopsy & 1 \\
\hline Hypersensitivity pneumonitis & $\begin{array}{l}\text { History } \\
\text { Blood gas analysis } \\
\text { HRCT } \\
\text { Respiratory function test }\end{array}$ & 1 \\
\hline Hemosiderosis & $\begin{array}{l}\text { Anemia } \\
\text { Serial chest } \\
\text { X-rays } \\
\text { Siderophages in gastric lavage }\end{array}$ & 1 \\
\hline Pulmonary lymphangiectasia & $\begin{array}{l}\text { HRCT } \\
\text { Pleural fluid }\end{array}$ & 1 \\
\hline Aspiration syndrome/mineral oil & $\begin{array}{l}\text { History } \\
\text { X-ray } \\
\text { BAL }\end{array}$ & 1 \\
\hline Aspiration syndrome/GER & $\begin{array}{l}\text { HRCT } \\
\text { pH-metry } \\
\text { Serial swallow study }\end{array}$ & 1 \\
\hline
\end{tabular}

$\mathrm{COP}=$ cryptogenic organizing pneumonia; DIP = desquamative interstitial pneumonia; $\mathrm{BAL}=$ bronchoalveolar lavage; LIP = lymphocytic interstitial pneumonia; NSIP = nonspecific interstitial pneumonia; GER = gastroesophageal reflux; HRCT = high resolution computerized tomography. 
with just two normal results. In three cases, we attempted monitoring outcome using 67-gallium scintigraphy, which was abnormal in one patient who continued the treatment.

Bronchoalveolar lavage in children requires sedation or anesthesia, its diagnostic capacity is limited in immunocompetent patients, ${ }^{22}$ and standardization for children is still under discussion. Technical recommendations for using BAL in children, normal values and areas for future studies were published in 2000 by the ERS task force. ${ }^{23}$ In our series, including critically ill and hypoxemic patients, when it was necessary to choose an invasive examination method, we indicated lung biopsy, since it offers more objective information, including on prognosis, with the ability to assess the degree of fibrosis. Nevertheless, BAL offers many possibilities for diagnosis ${ }^{24}$ and follow-up, depending on the health center where patients are being assessed, since many of the tests performed nowadays in developed countries are not yet available in clinical practice in our country. In our series, BAL contributed to the specific diagnosis in one case of aspiration of mineral oil (Table 3).

Biopsy is considered the diagnostic gold standard because it indicates the presence of interstitial inflammation, with alveolar wall thickening by various types of inflammatory cells and/or fibrosis. ${ }^{4,25}$ In our experience, this is the test that provides most information. The prognosis and treatment decisions in each case depend on a correlation between clinical, radiological and histopathological data. We always attempt to discuss diagnostic possibilities with the pathologists. We have found that biopsy findings with a predominance of inflammatory cells over degree of fibrosis are correlated with better response to treatment and better outcomes. Lung biopsy should be indicated early in cases where ILD is suspected, as soon as all noninvasive tests have been exhausted, even in critically ill patients, before pulmonary fibrosis sets in, which is an irreversible severity factor. 25,26 We only performed conventional histopathological tests, but recent research has made it possible to identify childhood-specific forms of ILD. So, we should be aware in the future of the need for specific tests, such as immunohistochemical assays, electronic microscopy and immunological techniques that analyze surfactant proteins. In a recent paper, Monaghan recommended that multiple biopsies should be performed. ${ }^{27}$

Hydroxychloroquine was used in six patients as a substitute drug or to reduce the dosage of corticosteroid. In one patient pulse therapy with corticosteroids was prescribed. The responses to antinflammatories vary, but at the present time the recommended treatment remains unaltered, with changes depending on patient response or in cases of significant side effects.

There is no consensus on treatment regimes for cases that progress to fibrosis. Several drugs are being tested, ${ }^{28,29}$ but we did not use them in any patient. Currently, a growing number of children at advanced ILD stages are undergoing lung transplantation, and survival is similar to those observed in other diseases. ${ }^{29,30}$

Patients with a good or regular outcome improved their physical and psychomotor development, important parameters in observational studies of pediatric patients.

In the majority of cases we managed to cultivate good compliance by patients' families with long-term therapy through explanations about the nature of the disease and making effort to develop a good patient-doctor relationship, fundamental when dealing with chronic patients.

Epidemiological research into interstitial lung diseases in children is made difficult by the scarcity of information. Because systematic studies in children have only recently been published, knowledge of these diseases in the pediatric age group is fragmented and their prevalence is unknown. Problems that have been identified as hindering better understanding are the lack of series with standardized diagnosis and treatment, and few reports of long-term follow-up. 5,19,20 During the period $1997-2002,{ }^{15}$ a group of pediatric pulmonologists from the ERS organized a task force which sent questionnaires to all European pneumology centers and assessed records from 185 patients. Clinical data and invasive and non-invasive supplementary tests made diagnosis possible in 177 (95.6\%) cases. In 67 patients that underwent lung biopsy, the diagnosis reported was not compatible with the standardized classification described above. Although the European study is the largest in the literature, its authors raised several questions and emphasized, as the main problem, the need to establish a classification of pediatric interstitial lung diseases. We do not know whether studies involving adult patients and animal models can be applied without restrictions to children, since they have specific immunological characteristics of defense and repair, in addition to their pulmonary development not being complete. Multicenter groups are being formed for prospective and retrospective studies aimed at better understanding of the problem and obtaining larger cohorts for treatment trials. ${ }^{5}$

A similar study is needed in Brazil and Latin America to investigate epidemiological, clinical, and outcome data.

\section{Acknowledgements}

We are grateful to the Pathology Service at the Hospital dos Servidores do Estado, in particularly to Dr. Francisca Gonçalves de Carvalho, Head of the Service, for her minutely detailed study of our patients' biopsies.

To Dr. Claudia Escosteguy, head of the Epidemiology Service of the Hospital dos Servidores do Estado, for her critical review of the article.

\section{References}

1. Fan LL, Mullen AL, Brugman SM, Inscore SC, Parks DP, White CW. Clinical spectrum of chronic interstitial lung disease in children. J Pediatr. 1992:121:867-72. 
2. Barbato A, Panizzolo C. Chronic interstitial lung disease in children. Paediatr Respir Rev. 2000;1:172-8.

3. Fauroux B, Epaud R, Clément A. Clinical presentation of interstitial lung disease in children. Pediatr Respir Rev. 2004; 5:98-100.

4. Clément A, Fauroux B. Mini-symposium: interstitial lung diseases in children. Paediatr Respir Rev. 2004;5:93.

5. Bush A. Paediatric interstitial lung disease: not just a kid's stuff. Eur Respir J. 2004;24:521-3.

6. American Thoracic Society; European Respiratory Society. American Thoracic Society/European Respiratory Society International Multidisciplinary Consensus Classification of Idiopathic Interstitial Pneumonias. This joint statement of the American Thoracic Society (ATS), and the European Respiratory Society (ERS) was adopted by the ATS board of directors, June 2001 and by ERS Executive Committee, June 2001. Am J Respir Crit Care Med. 2002;165:277-304.

7. Fan LL, Deterding RR, Langston C. Pediatric interstitial lung disease revisited. Pediatr Pulmonol. 2004;38:369-78.

8. Canakis AM, Cutz E, Manson D, O’Brodovich H. Pulmonary interstitial glycogenosis: a new variant of neonatal interstitial lung disease. Am J Respir Crit Care Med. 2002;165:1557-65.

9. Katzenstein AL, Gordon LP, Oliphant M, Swender PT. Chronic pneumonitis of infancy. A unique form of interstitial lung disease occurring in early childhood. Am J Surg Pathol. $1995 ; 19: 439-47$.

10. Deterding RR, Pye C, Fan LL, Langston C. Persistent tachypnea of infancy is associated with neuroendocrine cell hyperplasia. Pediatr Pulmonol. 2005;40:157-65.

11. Nogee LM. Genetic causes of surfactant deficiency. In: Chernick V, Boat TF, Wilmott RW, Bush A, editores. Kendig's disorders of the respiratory tract in children. Philadelphia: Saunders; 2006. p. 359-67.

12. Fan LL, Kozinetz CA. Factors influencing survival in children with chronic interstitial lung disease. Am J Respir Crit Care Med. 1997;156(3 Pt 1):939-42.

13. Fan LL, Kozinetz CA, Deterding RR, Brugman SM. Evaluation of a diagnostic approach to pediatric interstitial lung disease. Pediatrics. $1998 ; 101(1$ Pt 1):82-5.

14. Dinwiddie R. Treatment of interstitial lung disease in children. Paediatr Respir Rev. 2004;5:108-15.

15. Clement A; ERS Task Force. Task force on chronic interstitial lung disease in immunocompetent children. Eur Respir J. 2004;24:686-97.

16. Avital A, Godfrey S, Maayan C, Diamant Y, Springer C. Chloroquine treatment of interstitial lung disease in children. Pediatr Pulmonol. 1994;18:356-60.

17. Paul K, Klettke U, Moldenhauer J, Muller KM, Kleinau I, Magdorf $\mathrm{K}$, et al. Increasing dose of methylpredinisolone pulse therapy treats desquamative interstitial pneumonia in a child. Eur Respir J. $1999 ; 14: 1429-32$.
18. Balfour-Lynn IM, Primhak RA, Shaw BN. Home oxygen for children: who, how and when? Thorax. 2005;60:76-81.

19. Barbato A, Panizzolo C, Cracco A, de Blic J, Dinwiddie R, Zach M. Interstitial lung disease in children : a multicentre survey on diagnostic approach. Eur Respir J. 2000;16:509-13.

20. Dinwiddie R, Sharief N, Crawford O. Idiopathic interstitial pneumonitis in children: a national survey in the United Kingdom and Ireland. Pediatr Pulmonol. 2002;34:23-9.

21. Long FR, Castile RG. Technique and clinical applications of fullinflation and end-exhalation controlled-ventilation chest CT in infants and young children. Pediatr Radiol. 2001;31:413-22.

22. Fan LL, Lung MC, Wagener JS. The diagnostic value of bronchoalveolar lavage in immunocompetent children with chronic diffuse pulmonary infiltrates. Pediatr Pulmonol. 1997;23:8-13.

23. de Blic J, Midulla F, Barbato A, Clement A, Dab I, Eber E, et al. Bronchoalveolar lavage in children. ERS Task Force on bronchoalveolar lavage in children. European Respiratory Society. Eur Respir J. 2000;15:217-31.

24. Ronchetti R, Midulla F, Sandstrom T, Bjermer L, Zebrak J, Pawlik $\mathrm{J}$, et al. Bronchoalveolar lavage in children with chronic diffuse parenchymal lung disease. Pediatr Pulmonol. $1999 ; 27: 395-402$.

25. Katzenstein AL, Myers JL. Idiopathic pulmonary fibrosis: clinical relevance of pathologic classification. Am J Respir Crit Care Med. 1998;157(4 Pt 1):1301-15.

26. Hilman BC, Amaro-Galvez R. Diagnosis of interstitial lung disease in children. Paediatr Respir Rev. 2004;5:101-7.

27. Monaghan $H$, Wells AU, Colby TV, du Bois RM, Hansell DM, Nicholson AG. Prognostic implications of histologic patterns in multiple surgical lung biopsies from patients with idiopathic interstitial pneumonias. Chest. 2004;125:522-6.

28. Raghu G, Chang J. Idiopathic pulmonary fibrosis: current trends in management. Clin Chest Med. 2004;25:621-36.

29. Selman M. From anti-inflammatory drugs through antifibrotic agents to lung transplantation: a long road of research, clinical attempts, and failures in the treatment of idiopathic pulmonary fibrosis. Chest. 2002;122:759-61.

30. Mallory GB, Spray TL. Paediatric lung transplantation. Eur Respir J. $2004 ; 24: 839-45$.

Correspondence:

Maria Aparecida de Souza Paiva

Rua Voluntários da Pátria, 445/1101, Botafogo

CEP 22270-000 - Rio de Janeiro, RJ - Brazil

Tel.: +55 (21) 2539.0095

E-mail: mariaaparecida.paiva@gmail.com 